\title{
Factores personales y psicológicos que influyen en la Intención Emprendedora en estudiantes de la Universidad de Panamá
}

\section{Personal and Psychological Factors that Influence Entrepreneurial Intention in Students of the University of Panama}

Cleofé Genoveva Alvites-Huamaní* https://orcid.org/0000-0001-6328-6470

Omar López Sinisterra ${ }^{* *}$ https://orcid.org/0000-0002-4197-1212

Enrique Berra Ruiz ${ }^{* * *}$ https://orcid.org/0000-0003-3434-6264

Sandra Ivonne Muñoz Maldonado****https://orcid.org/0000-0002-9392-5023

http://dx.doi.org/10.21503/lex.v18i25.2112

* Coordinadora de Investigación, Vicerrectorado de Investigación, Innovación y Emprendimiento, Universidad Alas Peruanas.

Correo electrónico: cleoalvitesh@gmail.com

**Profesor Titular de la Universidad de Panamá.

Correo electrónico: tokuterada@yahoo.es

***Profesor Investigador Titular B de T.C. Escuela de Ciencias de la Salud de la Universidad Autónoma de Baja California, México.

Correo electrónico: eberrar@gmail.com

****Profesora Titular de la Facultad de Estudios Superiores Iztacala de la Universidad

Nacional Autónoma de México.

Correo electrónico: simmpsic@gmail.com

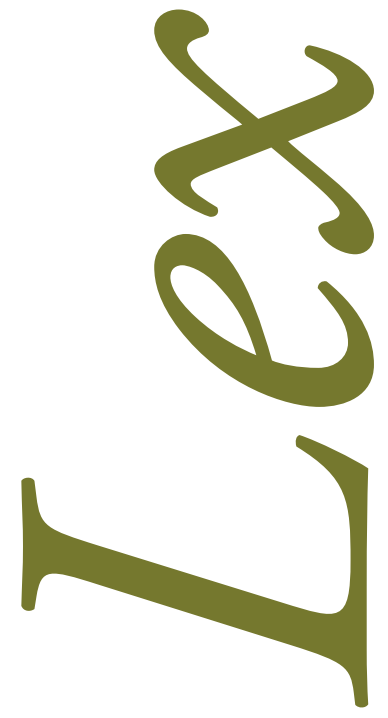




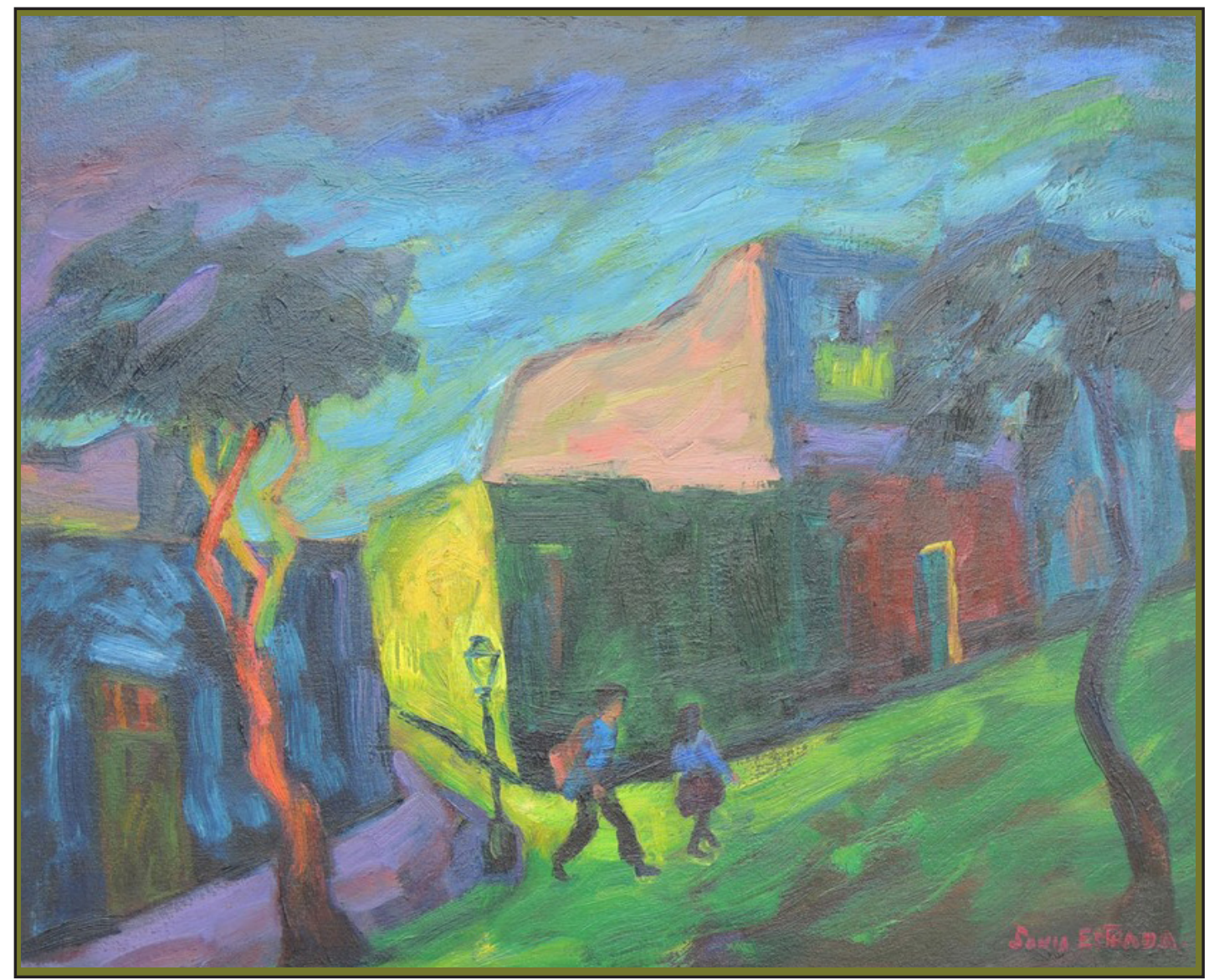

Barrios Altos. Óleos sobre lienzo 54 x $65 \mathrm{~cm}$. Sonia Estrada Melgarejo (pintora peruana, Ancash) 


\title{
RESUMEN
}

El objetivo del presente estudio fue analizar la relación explicativa de la intención emprendedora, mediante el análisis de las múltiples relaciones entre autoeficacia para el emprendimiento, el ámbito personal, formación para la creación de empresa, actitud para emprendimiento, motivación para la creación de empresa, apoyo financiero como factores personales de los estudiantes de pregrado de la Universidad de Panamá. Se aplicaron dos cuestionarios, en una muestra de 474 estudiantes, entre 16 y 60 ańos, del sexo femenino en mayor porcentaje, pertenecientes a las diferentes escuelas académicas. Se realizó el análisis bajo el modelo de ecuaciones estructurales para explicar las relaciones entre las variables de interés, para ello se identificaron cinco modelos, siendo el último modelo referido a la motivación, que resultó ser la variable que mejor predice y explica la intención emprendedora. Se discute el rol de la motivación en la intención emprendedora en contraposición a la actitud emprendedora que reporta la literatura.

Palabras Clave: emprendimiento, factores personales, psicológicos, motivación, autoeficacia.

\begin{abstract}
The objective of this study was to analyze the explanatory relationship of entrepreneurial intention, by analyzing the multiple relationships between self-efficacy for entrepreneurship, personal scope, training for the creation of a company, attitude for entrepreneurship, motivation for the creation of a company, financial support as personal factors of the undergraduate students of the University of Panama. Two questionnaires were applied, in a sample of 474 students, between 16 and 60 years old, of the female sex in a higher percentage, belonging to the different academic schools. Carrying out the analysis under the structural equations model to explain the relationships between the variables of interest, five models were identified for this, the last model referring to motivation, which turned out to be the variable that best predicts and explains intention enterprising. The role of motivation in entrepreneurial intention as opposed to the entrepreneurial attitude reported in the literature is discussed.
\end{abstract}

Key words: entrepreneurship, personal/psychological factors, motivation, self-efficacy. 


\section{INTRODUCCIÓN}

Los cambios derivados de la crisis económica mundial ha hecho que se replanteen perfiles de los futuros profesionales, haciendo que estos desarrollen competencias vitales para su inserción laboral, dejando de lado el enfocarse en cumplir un perfil vinculado directamente a un puesto en una empresa, debido a que ha surgido un fenómeno mundial que está en aumento como es el emprendimiento, considerado como una alternativa de desarrollo ante la crisis económica global y es visto como un motor de crecimiento para la creación de empresas, en las que están involucradas disciplinas como economía, historia, psicología y sociología que han considerado los elementos impulsores del emprendimiento y el perfil del emprendedor.

El estudio del emprendimiento es un aspecto muy complejo debido a que el proceso de creación de empresas implica varios elementos que sobrepasan una formación práctica en el análisis del mercado o una elaboración de planes de negocios, es necesario considerar que quien efectúa el proceso es un individuo con atributos personales, psicológicos, culturales, institucionales, políticos y hasta religiosos que influyen en su ejecución; Delgado-García, Rodríguez-Escudero y Martín-Cruz ${ }^{1}$; Alon$\mathrm{so}^{2}$, enmarcan que los rasgos afectivos y emocionales asumen un fuerte impacto en el desempeño del emprendedor, es por ello que una de las disciplinas que brinda mayor aporte a la comprensión del emprendedor como individuo es la psicología. Del Río ${ }^{3}$ refiere que la universidad también es fundamental, ya que ésta cumple un rol en la educación emprendedora, ya que debe brindar cursos que sirvan de iniciativa, apoyo y fomento en la intención emprendedora.

\section{EMPRENDIMIENTO}

Proviene del francés 'entrepreneur', que significa pionero y etimológicamente es la capacidad de una persona de realizar un esfuerzo adicional para alcanzar una meta, sin embargo, actualmente se emplea para referirse a la persona que inicia una nueva empresa o proyecto, con una actitud innovado-

1. Juan Bautista Delgado-García, Ana Isabel Rodríguez-Escudero, and Natalia Martín-Cruz, "Influence of affective traits on entrepreneur's goals and satisfaction”, Journal of Small Business Management 50.3 (2012): 408-428.

2. Patricia Esther Alonso Galicia, "La configuración de la intención emprendedora entre académicos responsables de proyectos de investigación en España. Un enfoque de género.” (2012).

3. Ainara Zubillaga Del Río, "El emprendimiento como elemento de formación universitaria: en busca de nuevas propuestas", Creatividad y sociedad: revista de la Asociación para la Creatividad 23 (2015): 182-199. 
ra, flexible, dinámica, capaz de asumir riesgos, creativo y orientado al crecimiento (Castillo ${ }^{4}$; Mavila, Tinoco \& Campos $^{5}$; Cabana-Villca, et al. ${ }^{6}$; Rodríguez ${ }^{7}$ ), lo que deriva al termino de emprendedor que para Moriano, Trejo \& Palaci ${ }^{8}$ es: "La persona que pone en marcha una iniciativa empresarial, crea su propia empresa, solo o asociado con otros promotores asumiendo los riesgos financieros que esto supone, aportando su trabajo y ocupándose de la dirección de la empresa”. La Comisión Europea refiere que es "la habilidad de la persona para transformar ideas en actos, vinculado a la creatividad, la innovación y asumir riesgos".

En concordancia con estas definiciones se puede identificar que la formación y desarrollo de nuevas empresas es un proceso complejo que implica tres factores: 1) las aspiraciones o motivaciones del creador, 2) sus competencias y recursos, 3) el ambiente o entorno para la creación, que se refiere a las oportunidades que ofrece el mercado y a los incentivos que brinda el marco legal (Marulanda, Montoya $\left.\& V^{\prime} e_{e}{ }^{9}\right)$. Dentro de estos elementos el papel principal lo tiene el individuo que busca crearla, ya que será quien deberá asumir la responsabilidad social desde su formación hasta la puesta en marcha, donde el liderazgo juega un papel trascendental para asegurar a largo plazo empresas viables y sostenibles socialmente $\left(\mathrm{Orrego}^{10}\right)$.

Con base en estos aspectos la literatura reporta estudios de elementos que intervienen en la creación de una empresa (Veciana ${ }^{11}$ ), como: 1) identificar una oportunidad empresarial, 2) los factores de producción, 3) el mercado en el que va a operar la nueva empresa, 4) la estrategia de combinación de recursos y de cómo llegar al mercado objetivo, 5) el empresario con una motivación, preparación y habilidades adecuadas para la toma de decisiones. En tanto que otras investigaciones hacen mayor referencia a las características individuales del emprendedor (Bosma ${ }^{12}$ ), en donde se plantean dos tipos de emprendimiento: 1) el de oportunidad, que se centra en el que las personas que perciben una oportunidad de negocio y crean empresas, 2) el de necesidad, que buscan las personas no porque quieran ser independientes sino porque no encuentran otra forma para subsistir.

4. Alicia Castillo, "Estado del arte en la enseñanza del emprendimiento", Emprendedores como creadores de riqueza y desarrollo regional 21 (1999).

5. Daniel Mavila Hinojoza, Óscar Tinoco Gómez, and César Campos Contreras, "Factores influyentes en la capacidad emprendedora de los alumnos de la Universidad Nacional Mayor de San Marcos”, Industrial Data 12,2 (2009): 3239.

6. Ricardo Cabana-Villca, Ivan Cortes-Castillo , Diego Plaza-Pasten, Mauricio Castillo-Vergara, \& Alejandro AlvarezMarin, "Análisis de las capacidades emprendedoras potenciales y efectivas en alumnos de centros de educación superior", Journal of technology management \& innovation 8,1 (2013): 65-75.

7. Fidel Rodriguez Batalla, "Estudio de la intención emprendedora en el ámbito científico público. El caso de las ciencias de la vida en España" (tesis doctoral, Universitat Politècnica de València, 2015).

8. Juan Antonio Moriano, Enrique Trejo, and Francisco J. Palací, "El perfil psicosocial del emprendedor: un estudio desde la perspectiva de los valores”, Revista de psicología Social 16,2 (2001): 229-242.

9. Flor Á. Marulanda Valencia, Iván A. Montoya Restrepo and Juan M. Vélez Restrepo, “Aportes teóricos y empíricos al estudio del emprendedor", Cuadernos de administración 30,51 (2014): 89-99.

10. Clara Inés Orrego Correas, "La dimensión humana del emprendimiento", Revista Ciencias Estratégicas 16.20 (2008): 225-236.

11. José M Veciana, La creación de empresas: Un enfoque gerencial. Caixa, Servicio de Estudios, 2005.

12. Niels Bosma, "The Global Entrepreneurship Monitor (GEM) and its impact on entrepreneurship research", Foundations and Trends in Entrepreneurship 9,2 (2013). 
Con respecto a la actitud emprendedora (Espíritu \& Sastre ${ }^{13}$; Espíritu ${ }^{14}$ ), se identifican los rasgos de personalidad como las cualidades, atributos, factores y hábitos que distinguen a una persona de las demás, estas características psicológicas internas determinan y reflejan la forma en que el individuo responde a su ambiente (Schiffman $\&$ Kanul $^{15}$; LópezSantos $\&$ Bueno $^{16}$; Shane, Locke \& Collins ${ }^{17}$; Shane \&Venkatara$\operatorname{man}^{18}$; McClelland ${ }^{19}$ ), reconociendo a la alta necesidad de logro, la toma de riesgos moderado, toma de decisión, responsabilidad personal, perseverancia, compromiso, liderazgo, tolerancia a cambios, percepción de beneficio económico, capacidad de análisis del ambiente/reflexión, como las más significativas.

Otras investigaciones (Cavazos-Arroyo, Puente-Díaz \& Ayup-González ${ }^{20}$; Rodríguez, 2015, Op.cit, 4p; Moriano, Trejo \& Palací, 2001, Op.cit, 4p; Schwartz \& Bilsky ${ }^{21}$ ) han centrado su interés en los valores y actitudes relacionadas con las necesidades de los emprendedores como los principios que guían su vida, de manera que el poder, logro, autodirección y estimulación, sean los valores que correlacionan de forma positiva y significativamente con el rol de emprendedor.

El ámbito personal según Durán-Aponte y Arias-Gómez ${ }^{22}$; Martínez-Rodríguez y Carmona ${ }^{23}$, debe considerarse como un aspecto relacionado con el ambiente familiar, estados emocionales, habilidades sociales, control emocional, empatía, entre otros; dentro del cual también se considera los rasgos

13. Roberto Espíritu Olmos, and Miguel Ángel Sastre Castillo, "La actitud emprendedora durante la vida académica de los estudiantes universitarios", Cuadernos de estudios empresariales 17 (2007): 95-116.

14. Roberto Espíritu Olmos, “Análisis de la intención emprendedora en estudiantes universitarios a través de los rasgos de personalidad", Multiciencias 11,1 (2011): 65-75.

15. Michael R. Solomon, and Miguel Angel Sanchez Carrion, Comportamiento del consumidor (Pearson, 2017)

16. Isidro de Pablo López, Begoña Santos Urda, and Yolanda Bueno Hernández. "Las dimensiones del perfil del emprendedor: contraste empírico con emprendedores de éxito." Presentado en el congreso El emprendedor innovador y la creación de empresas de I+ D+ I. Universidad de Valencia: Valencia, España. 2004.

17. Scott Shane and Sankaran Venkataraman, The promise of entrepreneurship as a field of research. (Berlín:Heidelberg, 2007), 171-184.

18. Scott Shane, Edwin A. Locke, and Christopher J. Collins, "Entrepreneurial motivation", Human resource management review 13,2 (2003): 257-279.

19. David Clarence McClelland, “Achievement-motivation can be developed.” Harvard business review 43,6 (1965): 6 .

20. Judith Cavazos-Arroyo, Rogelio Puente-Díaz, and Jannett Ayup-González, “Análisis de antecedentes de la intención al Emprendimiento social entre emprendedores potenciales de bajos ingresos en México". Vincula Tégica EFAN 2, 1 (2016): 189-1915.

21. Shalom H Schwartz and Wolfgang Bilsky, "Toward a theory of the universal content and structure of values: Extensions and cross-cultural replications", Journal of personality and social psychology 58,5 (1990): 878.

22. Emilse Durán-Aponte and Diana Arias-Gómez, "Intención emprendedora en estudiantes universitarios: integración de factores cognitivos y socio-personales." Revista Colombiana de Ciencias Sociales 6,2 (2015): 320-340.

23. Facundo Miguel Martínez-Rodríguez and Gabriel Carmona Orantes, "Test de factores sociopersonales para la inserción laboral de los jóvenes: un instrumento para la evaluación y la formación”, Estudios sobre educación 18 (2010): 115-138. 
de personalidad que no son obligatoriamente innatos, sino que pueden desarrollarse por el aprendizaje y la experiencia, lo que da pauta a considerar las capacidades y competencias, de esta manera se puede ubicar la creatividad, la disposición para asumir riesgos y la capacidad para solucionar problemas como características de los emprendedores (Penteado, Massukado-Nakatni \& Baron ${ }^{24}$; Moriano, Trejo \& Palací, 2001, Op.cit, 4p).

Un aspecto importante a considerar, es la percepción que tiene el emprendedor sobre sí mismo en relación con sus capacidades y habilidades, la cual está vinculada con la autoeficacia percibida, que implica que un individuo estará dispuesto a poner todo su empeño para desarrollar las actividades si considera que posee habilidades suficientes para hacerlo $\left(\right.$ Bandura $\left.^{25}\right)$, así un emprendedor realizará todos los esfuerzos para iniciar una empresa si considera que tiene las capacidades para que ésta sea exitosa, al confiar en su propias habilidades, en la percepción que tiene de oportunidades para emprender y el desempeñar tareas requeridas para crear una nueva empresa (Guerrero y Santamaría-Velasco $^{26}$; Sorondo ${ }^{27}$; Moriano, Montoya \& Vélez, 2001, Op.cit, 4p; Valbuena \& Borda ${ }^{28}$; Cernas-Ortiz, Mercado-Salgado \& Davis ${ }^{29}$ ), incorporando una actitud para emprender y la valoración de las consecuencias de emprender, (Aragón \& Baixaulii ${ }^{30}$ : Krauss $^{31}$; Castellanos, Latorre, Mateus \& Navarro ${ }^{32}$ ).

La motivación es otro elemento que contribuye a la comprensión de la conducta emprendedora debido a que todos los seres humanos estamos motivados por el deseo de alcanzar o mantener las condiciones con las que tengamos satisfechas las necesidades básicas que plantea Maslow (Shafritz et al. ${ }^{33}$ ), la motivación puede surgir de la desestabilización en la vida de las personas (Shapero ${ }^{34}$ ), lo que

24. José Pedro Penteado Pedroso, Márcia Shizue Massukado-Nakatani, and Fabrício Baron Mussi, "A relação entre o jeitinho brasileiro e o perfil empreendedor: possíveis interfaces no contexto da atividade empreendedora no Brasil", RAM. Revista de Administração Mackenzie 10,4 (2009): 100-130.

25. Albert Bandura, "Self-efficacy: toward a unifying theory of behavioral change." Psychological review 84,2 (1977): 191.

26. Maribel Guerrero y Carlos Alberto Santamaría-Velasco. "Ecosistema y actividad emprendedora en México: un análisis exploratorio", Perfiles Latinoamericanos, 28, 55 (2020): 227-251.

27. Julio Sorondo and M. E. Brenlla, "Locus de control y autoeficacia en emprendedores argentinos"." Trabajo de integración final, Universidad católica argentina (2011).

28. Pedro Nel Valbuena Hernández and Ricardo Alberto Borda Hernández, "Características emprendedoras en estudiantes de la Universidad El Bosque (Colombia)", Revista Espacios 38,30 (2017).

29. Daniel Arturo Cernas-Ortiz, Patricia Mercado-Salgado and Mark A. Davis, "Perspectiva futura de tiempo, satisfacción laboral y compromiso organizacional: el efecto mediador de la autoeficacia, la esperanza y la vitalidad." Revista de Psicología del Trabajo y de las Organizaciones 34,1 (2018): 1-9.

30. A. Aragón, \& S. Baixauli, Intención Emprendedora de los estudiantes de Bachillerato y Ciclos Formativos en la Región de Murcia (Universidad de Murcia, 2014).

31. Catherine Krauss, "Actitudes emprendedoras de los estudiantes universitarios: El caso de la Universidad Católica del Uruguay”, Dimensión empresarial 9, 1 (2011): 28-40.

32. Virgelina Castellanos Páez, et al., "Modelo explicativo del desempeño académico desde la autoeficacia y los problemas de conducta", Revista Colombiana de psicología 26,1 (2017): 149-161.

33. Jay M. Shafritz, J. Steven Ott, and Yong Suk Jang, Classics of organization theory (Cengage:Learning, 2015).

34. Albert Shapero, "Why entrepreneurship? A worldwide perspective." Journal of Small Business Management (pre1986) 23.000004 (1985): 1. 
puede generar un emprendimiento por conveniencia o la deseabilidad y por factibilidad o viabilidad, las primeras se basan en los valores, mientras que la factibilidad se centra en la educación formal, recursos y experiencia obtenida.

Estos dos planteamientos integrados permiten ser abordados en la teoría del comportamiento planificado de Ajzen ${ }^{35}$, bajo tres conceptos explícitos 1) la actitud hacia el comportamiento, en donde una persona cuenta con una valoración favorable o desfavorable de la conducta en cuestión, en este caso ser emprendedor, 2) la norma subjetiva, en donde se considera la percepción de la presión social para realizar la conducta, 3) el grado de control conductual percibido, relacionado con la facilidad o dificultad percibida de realizar la conducta, (Rueda, Hernández \& Herrero $^{36}$ ). Asimismo la motivación (Paredes ${ }^{37}$; Cordero, Astudillo, Carpio, Delgado \& Amón ${ }^{38}$; Raposo, Paco \& Ferreira ${ }^{39}$; López, Santos \& Bueno, 2004, Op.cit, 5p; Kirkwood \& Brown ${ }^{40}$ ) ha brindado mayor certeza para la consolidación de un emprendedor, dado que se requiere de una gran cantidad de esfuerzo, donde el deseo de independencia, autonomía, ganar más dinero, satisfacción en el trabajo, tener un desafío, ser su propio jefe, tener un estatus, son variables constantes en sus conductas para lograr sus aspiraciones y crear una nueva empresa.

Las características identificadas entre los emprendedores, se relacionan con comportamientos que pueden modificarse o aprenderse más que con aspectos de personalidad (Burgos, Pomboza \& Villar ${ }^{41}$; Zabala ${ }^{42}$; Amit $\&$ Muller $^{43}$ ), lo que fortalece la visión que el emprendimiento debe ser parte del proceso de educación de los jóvenes implementándolo en el currículo de los últimos semestres de algunas carreras universitarias.

Según Espino \& Vásquez ${ }^{44}$ desde la universidad se puede incentivar a que los estudiantes se conviertan en emprendedores exitosos; sin embargo, existen estudios (Espíritu, 2011, Op.cit, 5p; Urbano

35. Icek Azjen, “The theory of planned behavior", Organizational behavior and human decision processes 50,2 (1991): 179-211.

36. María Inés Rueda Sampedro, Ana Fernández Laviada, and Angel Herrero Crespo, "Aplicación de la teoría de la acción razonada al ámbito emprendedor en un contexto universitario", (2013).

37. Maria Cristina Paredes Juárez, "Relación entre intención emprendedora, aspiraciones y satisfacción con la vida en universitarios limeños", Universidad Peruana de Ciencias Aplicadas. Lima-Perú (2015).

38. Juan Cordero López, et al., “Análisis de los factores que influyen el emprendimiento y la sostenibilidad de las empresas del área urbana de la ciudad de Cuenca, Ecuador”, Maskana 2,2 (2011): 27-37.

39. George Solomon, et al., "Entrepreneur's profile: a taxonomy of attributes and motivations of university students", Journal of Small Business and enterprise development (2008).

40. J. Kirkwood and K. Brown, "Defining our entrepreneurs", NZ Business 15,10 (2001): 22-24.

41. R. C. Burgos, "Los emprendimientos como elemento de estudio teórico, académico e investigativo", Contribuciones a las Ciencias Sociales, Málaga, España, disponible en www. eumed.net/rev/cccss (2017).

42. R. Zabala, "Emprendimiento: un proceso de aprendizaje", (2013). Recuperado de http://www.madrimasd.org/ informacionIdi/analisis/opinion/opinion.asp?id=55885

43. R. Amit and E. Muller, "Contrasting attributes and attitudes on entrepreneurs and nonentrepreneurs", Academy of Management National Meeting. 1994.

44. Samuel Vásquez, "Intención emprendedora en estudiantes universitarios de Ingeniería Industrial Administrativa de la Universidad Católica Santa María la Antigua (USMA)", Investigación y Pensamiento Crítico 3,3 (2015): 1326. 
$\&$ Toledano ${ }^{45}$; Vesper \& McMullan $^{46}$ ) que han reportado que el cursar un programa de emprendimiento no convierte al participante en emprendedor, pero si se observan cambios significativos en su conocimiento para dicho proceso, como es el caso en la administración de un nuevo negocio, habilidades para identificar y evaluar oportunidades de negocios, definir la propuesta de valor, modelo del negocio, comunicar efectivamente sus ideas de negocio, construir su red de contactos y armar un buen equipo de trabajo, (Izquierdo ${ }^{47}$ ).

Burgos, Pomboza \& Villar (2008, Op.cit, 8p) indican que la preparación implica la asimilación de conocimientos que posibiliten la comprensión de su entorno para el aprovechamiento de los recursos y elementos que permiten orientar las cualidades hacia el desarrollo de su potencialidad emprendedora y promover cualidades psicológicas favorables para la actividad emprendedora como autoconfianza, autoestima, autoeficacia, necesidad de logro, etc. $\left(\right.$ Rasheed $\left.^{48}\right)$, derivando que la gran mayoría de los estudios se centren en estudiantes universitarios identificados como la población con mayor motor de crecimiento e innovación, con el fin de desarrollar capacidades para generar nuevos conocimientos, adoptar y adaptar mejores prácticas empresariales que puedan incorporarlas en programas de incubación de empresas o bien que brinden alternativas para atender las demandas industriales y de servicios.

Revisados estos enfoques se consideró como objetivo identificar la relación explicativa de la intención emprendedora, mediante el análisis de las múltiples relaciones entre autoeficacia para el emprendimiento, el ámbito personal, formación para la creación de empresa, actitud para emprendimiento, motivación para la creación de empresa, apoyo financiero como factores personales de los estudiantes de pregrado de la Universidad de Panamá.

\section{MATERIALES Y MÉTODO}

La muestra estuvo constituida por 474 estudiantes de 26 carreras distintas (tabla1) de la Universidad de Panamá, de los cuales el 30.8\% eran varones y el 69.2\% mujeres. El $86.1 \%$ son solteros, el $6.1 \%$ están en unión libre y el 7.8\% restante es casado. La edad osciló entre 16 y 60 años, teniendo una predominancia los jóvenes de entre 16 y 30 años.

45. David Urbano and Nuria Toledano Garrido, "Los sistemas de formación universitaria y su influencia en las actitudes empresariales de los estudiantes: Un estudio de casos múltiple”, Oikos: Revista de la Escuela de Administración y Economía 25 (2008): 5.

46. Robert Ronstadt, Karl H. Vesper and W. Ed McMullan, “Entrepreneurship: today courses, tomorrow degrees?”, Entrepreneurship theory and practice 13.1 (1988): 7-13.

47. Edgar Izquierdo, Edgar, Impact assessment of an educational intervention based on the constructivist paradigm on the development of entrepreneurial competencies in university students, (tesis doctoral, Ghent University, 2008).

48. Howard, S. Rasheed, Developing entrepreneurial potential in Youth: The effects of entrepreneurial education and venture creation. University of South Florida (2000). 
Tabla 1. Frecuencias por especialidad, en relación con el sexo, edad y estado civil

\begin{tabular}{|c|c|c|c|c|c|c|c|c|c|c|}
\hline \multirow[t]{3}{*}{ Especialidad } & \multicolumn{10}{|c|}{ Variables } \\
\hline & \multicolumn{2}{|c|}{ Sexo } & \multicolumn{5}{|c|}{ Edad } & \multicolumn{3}{|c|}{ Estado civil } \\
\hline & Hombres & Mujeres & $\begin{array}{l}16- \\
20\end{array}$ & $\begin{array}{l}21- \\
30\end{array}$ & $\begin{array}{l}31- \\
40\end{array}$ & $\begin{array}{l}41- \\
50\end{array}$ & $>50$ & Soltero & Unido & Casado \\
\hline Psicología & 0 & 2 & 0 & 2 & 0 & 0 & 0 & 1 & 0 & 1 \\
\hline $\begin{array}{l}\text { Psicología } \\
\text { clínica }\end{array}$ & 0 & 4 & 0 & 3 & 0 & 0 & 1 & 1 & 1 & 2 \\
\hline Farmacia & 2 & 9 & 0 & 11 & 0 & 0 & 0 & 10 & 1 & 0 \\
\hline $\begin{array}{l}\text { Administración } \\
\text { pública } \\
\text { aduanas }\end{array}$ & 13 & 39 & 44 & 7 & 0 & 0 & 1 & 50 & 2 & 0 \\
\hline $\begin{array}{c}\text { Lic. en } \\
\text { Humanidades }\end{array}$ & 1 & 1 & 1 & 0 & 0 & 1 & 0 & 1 & 0 & 1 \\
\hline Lic. en Inglés & 4 & 12 & 4 & 5 & 4 & 3 & 0 & 10 & 0 & 6 \\
\hline $\begin{array}{l}\text { Lic. en } \\
\text { Turismo } \\
\text { geográfico }\end{array}$ & 4 & 11 & 1 & 14 & 0 & 0 & 0 & 9 & 3 & 3 \\
\hline $\begin{array}{c}\text { Administración } \\
\text { de empresas }\end{array}$ & 5 & 26 & 19 & 9 & 3 & 0 & 0 & 27 & 3 & 1 \\
\hline $\begin{array}{l}\text { Medicina } \\
\text { Veterinaria }\end{array}$ & 18 & 37 & 34 & 21 & 0 & 0 & 0 & 54 & 0 & 1 \\
\hline Cirugía Dental & 9 & 26 & 1 & 34 & 0 & 0 & 0 & 34 & 1 & 0 \\
\hline Artes Visuales & 5 & 11 & 10 & 6 & 0 & 0 & 0 & 15 & 1 & 0 \\
\hline Ingeniería Civil & 3 & 5 & 7 & 1 & 0 & 0 & 0 & 8 & 0 & 0 \\
\hline $\begin{array}{l}\text { Turismo } \\
\text { Bilingüe }\end{array}$ & 12 & 30 & 29 & 13 & 0 & 0 & 0 & 37 & 3 & 2 \\
\hline Informática & 46 & 48 & 27 & 58 & 6 & 3 & 0 & 75 & 6 & 13 \\
\hline Ingeniería & 8 & 23 & 26 & 5 & 0 & 0 & 0 & 30 & 1 & 0 \\
\hline $\begin{array}{l}\text { Lic. en } \\
\text { Geografía }\end{array}$ & 5 & 4 & 5 & 3 & 1 & 0 & 0 & 9 & 0 & 0 \\
\hline Lic. en Español & 1 & 12 & 3 & 4 & 5 & 0 & 1 & 7 & 4 & 2 \\
\hline Sistemas & 1 & 0 & 0 & 1 & 0 & 0 & 0 & 1 & 0 & 0 \\
\hline Mercadeo & 0 & 2 & 1 & 1 & 0 & 0 & 0 & 2 & 0 & 0 \\
\hline
\end{tabular}




\begin{tabular}{ccccccccccc}
\hline $\begin{array}{c}\text { Educación } \\
\text { primaria }\end{array}$ & 1 & 0 & 0 & 1 & 0 & 0 & 0 & 1 & 0 & 0 \\
$\begin{array}{c}\text { Contabilidad } \\
\text { Lic. en }\end{array}$ & 3 & 7 & 0 & 8 & 2 & 0 & 0 & 6 & 1 & 3 \\
$\begin{array}{c}\text { Recursos } \\
\text { Humanos }\end{array}$ & 1 & 15 & 9 & 7 & 0 & 0 & 0 & 15 & 0 & 1 \\
$\begin{array}{c}\text { Lic. en } \\
\text { Derecho }\end{array}$ & 4 & 2 & 2 & 1 & 0 & 2 & 1 & 3 & 2 & 1 \\
$\begin{array}{c}\text { Forense } \\
\text { Administración }\end{array}$ & 0 & 1 & 0 & 1 & 0 & 0 & 0 & 1 & 0 & 0 \\
de Gerencia & 1 & 1 & 0 & 1 & 0 & 0 & 0 & 1 & 0 & 0 \\
$\quad$ Total & 146 & 328 & 223 & 217 & 21 & 9 & 4 & 408 & 29 & 37 \\
\hline
\end{tabular}

Fuente: Elaboración propia

Con respecto al ciclo escolar y especialidad que se cursa (tabla 2), la mayor proporción corresponden al segundo semestre (66.7\%), existen pocos casos de semestres avanzados y los que han respondido de octavo semestre en adelante corresponden a cirujanos dentistas, psicólogos clínicos, farmaceúticos y médicos veterinarios.

Tabla 2. Frecuencias por especialidad en relación con el ciclo de estudios

\begin{tabular}{|c|c|c|c|c|c|c|c|c|c|}
\hline \multirow[t]{2}{*}{ Variable } & \multicolumn{9}{|c|}{ Ciclo escolar } \\
\hline & I & II & III & IV & VI & VII & VIII & $\mathrm{X}$ & $\overline{\mathrm{XII}}$ \\
\hline Psicología & 0 & 1 & 0 & 0 & 1 & 0 & 0 & 0 & 0 \\
\hline Psicología clínica & 0 & 2 & 0 & 1 & 0 & 0 & 0 & 0 & 1 \\
\hline Farmacia & 0 & 6 & 0 & 0 & 0 & 3 & 2 & 0 & 0 \\
\hline $\begin{array}{l}\text { Administración pública } \\
\text { aduanas }\end{array}$ & 7 & 44 & 1 & 0 & 0 & 0 & 0 & 0 & 0 \\
\hline Lic. en Humanidades & 0 & 2 & 0 & 0 & 0 & 0 & 0 & 0 & 0 \\
\hline Lic. en Inglés & 0 & 16 & 0 & 0 & 0 & 0 & 0 & 0 & 0 \\
\hline Lic. en Turismo geográfico & 0 & 15 & 0 & 0 & 0 & 0 & 0 & 0 & 0 \\
\hline Administración de empresas & 0 & 30 & 0 & 0 & 0 & 0 & 0 & 0 & 0 \\
\hline Medicina Veterinaria & 0 & 17 & 2 & 31 & 2 & 0 & 1 & 2 & 0 \\
\hline Cirugía Dental & 0 & 1 & 0 & 7 & 1 & 0 & 26 & 0 & 0 \\
\hline Artes Visuales & 0 & 15 & 1 & 0 & 0 & 0 & 0 & 0 & 0 \\
\hline Ingeniería Civil & 0 & 0 & 8 & 0 & 0 & 0 & 0 & 0 & 0 \\
\hline Turismo Bilingüe & 1 & 41 & 0 & 0 & 0 & 0 & 0 & 0 & 0 \\
\hline Informática & 13 & 71 & 5 & 5 & 0 & 0 & 0 & 0 & 0 \\
\hline
\end{tabular}




\begin{tabular}{llllllllll} 
Ingeniería & 1 & 5 & 24 & 0 & 1 & 0 & 0 & 0 & 0 \\
Lic. en Geografía & 0 & 7 & 0 & 2 & 0 & 0 & 0 & 0 & 0 \\
Lic. en Espańol & 0 & 9 & 1 & 2 & 0 & 0 & 1 & 0 & 0 \\
Sistemas & 0 & 1 & 0 & 0 & 0 & 0 & 0 & 0 & 0 \\
Mercadeo & 1 & 1 & 0 & 0 & 0 & 0 & 0 & 0 & 0 \\
Educación primaria & 0 & 1 & 0 & 0 & 0 & 0 & 0 & 0 & 0 \\
Contabilidad & 0 & 8 & 0 & 2 & 0 & 0 & 0 & 0 & 0 \\
Lic. en Recursos Humanos & 0 & 16 & 0 & 0 & 0 & 0 & 0 & 0 & 0 \\
Lic. en Derecho & 0 & 6 & 0 & 0 & 0 & 0 & 0 & 0 & 0 \\
Forense & 0 & 0 & 0 & 0 & 0 & 0 & 1 & 0 & 0 \\
Administración de Gerencia & 0 & 1 & 0 & 0 & 0 & 0 & 0 & 0 & 0 \\
Total & 23 & 316 & 42 & 50 & 5 & 3 & 31 & 2 & 1 \\
Porcentaje & 4.9 & 66.7 & 8.9 & 10.6 & 1.1 & .6 & 6.6 & .4 & .2 \\
\hline
\end{tabular}

Fuente: Elaboración propia

Se indagó si estaban trabajando actualmente, sólo el 23.8\% reportó que sí trabajaba, el 6.3\% lo hace en una institución pública, $17.1 \%$ en empresa privada, y sólo un 3\% en una empresa propia. La experiencia laboral reportada es de 1 año para el 10.8\%, sólo un 5.1\% reporta de 2 a 3 años de trabajo, un $7 \%$ de 4 a 10 años y un $2.5 \%$ ha laborado por más de 10 años.

En la tabla 3 se observa que la mayor parte de ellos no ha laborado antes, la especialidad que reporta más casos que han laborado es la de informática, con uno o dos años de antigüedad, lo que indica, que desde que iniciaron sus estudios comenzaron a trabajar en empresas privadas.

Tabla 3. Frecuencias por especialidad en relación con su actividad laboral

\begin{tabular}{|c|c|c|c|c|c|c|c|c|c|c|c|}
\hline \multirow[t]{3}{*}{ Variable } & \multicolumn{11}{|c|}{ Variables } \\
\hline & \multicolumn{2}{|c|}{ Trabaja } & \multicolumn{5}{|c|}{ Desde hace cuánto } & \multicolumn{4}{|c|}{ Tipo de empresa en que labora } \\
\hline & SI & $\mathrm{NO}$ & 0 & $\begin{array}{c}1 \\
\text { año }\end{array}$ & $\begin{array}{c}2- \\
\text { 3años }\end{array}$ & $\begin{array}{l}4-10 \\
\text { años }\end{array}$ & $>10$ & Publica & Privada & Ninguna & Propia \\
\hline Psicología & 1 & 1 & 0 & 1 & 0 & 1 & 0 & 0 & 2 & 0 & 0 \\
\hline Psicología clínica & 4 & 0 & 0 & 0 & 3 & 0 & 1 & 2 & 2 & 0 & 0 \\
\hline Farmacia & 2 & 9 & 7 & 2 & 0 & 2 & 0 & 1 & 3 & 7 & 0 \\
\hline $\begin{array}{l}\text { Administración } \\
\text { pública aduanas }\end{array}$ & 6 & 46 & 44 & 7 & 0 & 0 & 1 & 0 & 8 & 44 & 0 \\
\hline $\begin{array}{c}\text { Lic. en } \\
\text { Humanidades }\end{array}$ & 1 & 1 & 1 & 1 & 0 & 0 & 0 & 0 & 1 & 1 & 0 \\
\hline Lic. en Inglés & 3 & 13 & 12 & 0 & 1 & 3 & 0 & 1 & 3 & 12 & 0 \\
\hline $\begin{array}{l}\text { Lic. en Turismo } \\
\text { geográfico }\end{array}$ & 3 & 12 & 11 & 2 & 1 & 1 & 0 & 0 & 3 & 11 & 1 \\
\hline
\end{tabular}




\begin{tabular}{|c|c|c|c|c|c|c|c|c|c|c|c|}
\hline $\begin{array}{c}\text { Administración de } \\
\text { empresas }\end{array}$ & 10 & 21 & 21 & 4 & 2 & 4 & 0 & 2 & 8 & 21 & 0 \\
\hline $\begin{array}{c}\text { Medicina } \\
\text { Veterinaria }\end{array}$ & 2 & 53 & 54 & 0 & 1 & 0 & 0 & 0 & 2 & 53 & 0 \\
\hline Cirugía Dental & 5 & 30 & 31 & 2 & 1 & 1 & 0 & 0 & 5 & 30 & 0 \\
\hline Artes Visuales & 1 & 15 & 12 & 4 & 0 & 0 & 0 & 1 & 3 & 11 & 1 \\
\hline Ingeniería Civil & 2 & 6 & 6 & 1 & 0 & 1 & 0 & 0 & 0 & 6 & 2 \\
\hline Turismo Bilingüe & 4 & 38 & 37 & 4 & 0 & 1 & 0 & 0 & 5 & 37 & 0 \\
\hline Informática & 42 & 52 & 52 & 17 & 14 & 6 & 5 & 14 & 22 & 52 & 6 \\
\hline Ingeniería & 3 & 28 & 29 & 1 & 0 & 1 & 0 & 0 & 2 & 28 & 1 \\
\hline Lic. en Geografía & 0 & 9 & 9 & 0 & 0 & 0 & 0 & 0 & 0 & 9 & 0 \\
\hline Lic. en Español & 8 & 5 & 5 & 1 & 0 & 6 & 1 & 3 & 5 & 5 & 0 \\
\hline Sistemas & 1 & 0 & 0 & 0 & 0 & 1 & 0 & 1 & 0 & 0 & 0 \\
\hline Mercadeo & 1 & 1 & 1 & 1 & 0 & 0 & 0 & 1 & 0 & 1 & 0 \\
\hline Educación primaria & 1 & 0 & 0 & 1 & 0 & 0 & 0 & 0 & 1 & 0 & 0 \\
\hline Contabilidad & 6 & 4 & 4 & 0 & 1 & 3 & 2 & 2 & 4 & 4 & 0 \\
\hline $\begin{array}{l}\text { Lic. en Recursos } \\
\text { Humanos }\end{array}$ & 0 & 16 & 16 & 0 & 0 & 0 & 0 & 0 & 0 & 16 & 0 \\
\hline Lic. en Derecho & 5 & 1 & 2 & 1 & 0 & 1 & 2 & 2 & 0 & 1 & 3 \\
\hline Forense & 1 & 0 & 0 & 0 & 0 & 1 & 0 & 0 & 1 & 0 & 0 \\
\hline $\begin{array}{c}\text { Administración de } \\
\text { Gerencia }\end{array}$ & 1 & 0 & 0 & 1 & 0 & 0 & 0 & 0 & 1 & 0 & 0 \\
\hline Total & 113 & 361 & 354 & 51 & 24 & 33 & 12 & 30 & 81 & 349 & 14 \\
\hline
\end{tabular}

Fuente: Elaboración propia

Para la recolección de datos se utilizaron dos instrumentos de elaboración propia que se describen a continuación:

Variable 1: Factores personales y psicológicos, consta de 117 ítems, con respuestas en escala tipo Likert de 5 opciones, que miden 6 dimensiones: Autoeficacia para emprendimiento, ámbito personal, formación para la creación de empresa, actitud para emprendimiento, motivación para la creación de empresa y apoyo financiero. 
Variable 2: Intención emprendedora, consta de 35 ítems, con respuestas en escala tipo Likert de 5 opciones, que miden 2 dimensiones: Emprendimiento independiente y emprendimiento corporativo.

La validez de ambos instrumentos fue realizada por tres expertos en la temática de la investigación, uno de Perú, dos de México y uno de Colombia, quienes indicaron que el instrumento era aplicable para la investigación.

La confiabilidad se analizó con el Alfa de Cronbach, los coeficientes de las escalas de autoeficacia, factores personales, formación para la empresa, apoyo financiero y motivación son aceptables de acuerdo con el criterio de Oviedo y $\mathrm{Arias}^{49}$, ya que se encuentran entre .70 y .90, sin embargo, las escalas de actitud para el emprendimiento, así como el emprendimiento independiente y corporativo presentan redundancia por tener un valor por encima de .90 (ver tabla 4).

Tabla 4. Descriptivos Alfa Cronbach de escalas de Factores personales y psicológicos e intención emprendedora

\begin{tabular}{lc}
\hline Factores personales y psicológicos & Alfa de Cronbach \\
\hline Autoeficacia para el emprendimiento & .891 \\
Factores personales & .815 \\
\hline Formación para la creación de empresa & .896 \\
\hline Actitud para emprendimiento & .932 \\
Motivación para la creación de empresa & .872 \\
\hline Apoyo financiero & .803 \\
\hline Intención Emprendedora & .937 \\
\hline Emprendimiendo independiente & .928 \\
\hline Emprendimiento corporativo & \\
\hline
\end{tabular}

Fuente: Elaboración propia

El análisis estadístico se realizó con el modelo de ecuaciones estructurales (SEM), técnica que emplea la regresión múltiple, el análisis factorial y permite emplear múltiples medidas que representan el constructo y controlar el error de medición especifico de cada variable, Cupani ${ }^{50}$.

49. Heidi Celina, Oviedo and Campo-Arias, Adalberto. "Aproximación al uso del coeficiente alfa de Cronbach", Revista Colombiana de Psiquiatría 34,4 (2005): 572-580.

50. Marcos Cupani, “Análisis de ecuaciones estructurales: conceptos, etapas de desarrollo y un ejemplo de aplicación”. Revista Tesis 1, (2012): 186-199. 
Para realizar el estudio se procedió a establer contacto con la dirección de los Centros de Innovación Desarrollo Tecnológico y Emprendimiento (CIDETE) de la Universidad de Panamá, con quien se coordinó para realizar la investigación. Posteriormente se solicitó la autorización a las escuelas académicas para que sus estudiantes participen del estudio.

Obtenida la autorización se aplico los cuestionaros durante una semana, previo consentimiento de cada estudiante, a quienes se les informó al momento de la aplicación la forma de responder y el tiempo requerido, al ser este voluntario y anónimo, el cual siguió los lineamientos indicados en el Manual de publicaciones APA ${ }^{51}$.

\section{RESULTADOS}

Respecto a los descriptivos de cada variable y su dimensión (tabla 5) pueden ver las puntuaciones medias en cada variable, siendo los puntajes actitud para el emprendimiento y el emprendimiento los que tuvieron puntuaciones altas.

Tabla 5. Descriptivos de las variables evaluadas

\begin{tabular}{lccc}
\multicolumn{1}{c}{ Variables } & $\begin{array}{c}\text { Intérvalo de } \\
\text { puntuaciones }\end{array}$ & Media & $\begin{array}{c}\text { Desviación } \\
\text { estándar }\end{array}$ \\
\hline Autoeficacia para el emprendimiento & $25-125$ & 86.2 & 13.6 \\
Factores personales & $27-135$ & 93.7 & 13.2 \\
\hline Formación para la creación de empresa & $13-65$ & 44.2 & 10.4 \\
\hline Actitud para emprendimiento & $40-200$ & 147.7 & 22.8 \\
\hline Motivación para la creación de empresa & $9-45$ & 31.1 & 5.6 \\
\hline Apoyo financiero & $3-15$ & 11.8 & 2.5 \\
\hline Emprendimiento independiente & $21-105$ & 80.46 & 15.36 \\
\hline Emprendimiento corporativo & $14-70$ & 52.51 & 11.48 \\
\hline
\end{tabular}

Fuente: Elaboración propia

51. APA - American Psycgological Association. Manual de Publicaciones de la American Psychological Association. (3ra. Ed.) México: El Manual Moderno, (2010). 
De acuerdo con el objetivo planteado se buscó obtener un modelo a través de ecuaciones estructurales para explicar las relaciones entre las variables de interés, para ello se identificaron 5 modelos de los cuales los modelos 4 y 5 explican mejor la relación de las variables con la diferencia de que en el último modelo el valor del RMR que se asocia con un mejor nivel de ajuste del modelo. En la tabla 6 se pueden identificar los índices de ajuste de cada modelo probado, tanto los de ajuste absoluto, como de ajuste incremental.

Tabla 6 Índices de ajuste de los modelos probados

\begin{tabular}{llllllllllllll}
\hline & & \multicolumn{4}{c}{ Índices de ajuste Absoluto } & \multicolumn{7}{c}{ Índices de ajuste incremental } \\
\hline Modelo & $\mathrm{X}^{2}$ & $\mathrm{gl}$ & $\mathrm{X} / \mathrm{gl}$ & GFI & AGFI & RMR & RMSEA & NFI & TLI & CFI & IFI \\
\hline 1 & 651.26 & 13 & 50.097 & .753 & .316 & 57.170 & .322 & .698 & .354 & .700 & .702 \\
\hline 2 & 520.67 & 7 & 74.381 & .745 & .235 & 57.59 & .394 & .691 & .341 & .693 & .694 \\
\hline 3 & 207.70 & 3 & 69.23 & .875 & .377 & 58.312 & .380 & .841 & .474 & .842 & .843 \\
\hline 4 & .222 & 1 & .222 & 1.000 & .998 & .479 & .000 & 1.000 & 1.004 & 1.000 & 1.001 \\
5 & .021 & 1 & .021 & 1.000 & 1.000 & .038 & .000 & 1.000 & 1.007 & 1.000 & 1.001 \\
\hline
\end{tabular}

Nota: gl: grados de libertad. GFI: Goodness of Fit Index (índice de bondad del ajuste). AGFI: Adjusted Goodness of Fit Index (índice de bondad de ajuste corregido). RMR: Root Mean Square Residual (residuo cuadrático medio). RMSEA: Root Mean Square Error of Approximation (error de aproximación cuadrático medio). NFI: Normed Fit Index (índice de ajuste normalizado). TLI: Tucker-Lewis coefficient (coeficiente de Tucker y Lewis). CFI: Comparative Fit Index (índice de ajuste comparativo). iIFI: Incremental Fit Index (índice de ajuste incremental).

El primer modelo probado (figura 1) incluyó todas las variables medidas y como variable latente la intención emprendedora con dos medidas (emprendimiento independiente y emprendimiento corporativo), sin embargo, los resultados de los indicadores además de mostrar poco ajuste, también sugerían que dos variables debían quitarse del modelo para mejorar el ajuste, dichas variables fueron el ámbito personal y la formación para creación de empresas. 


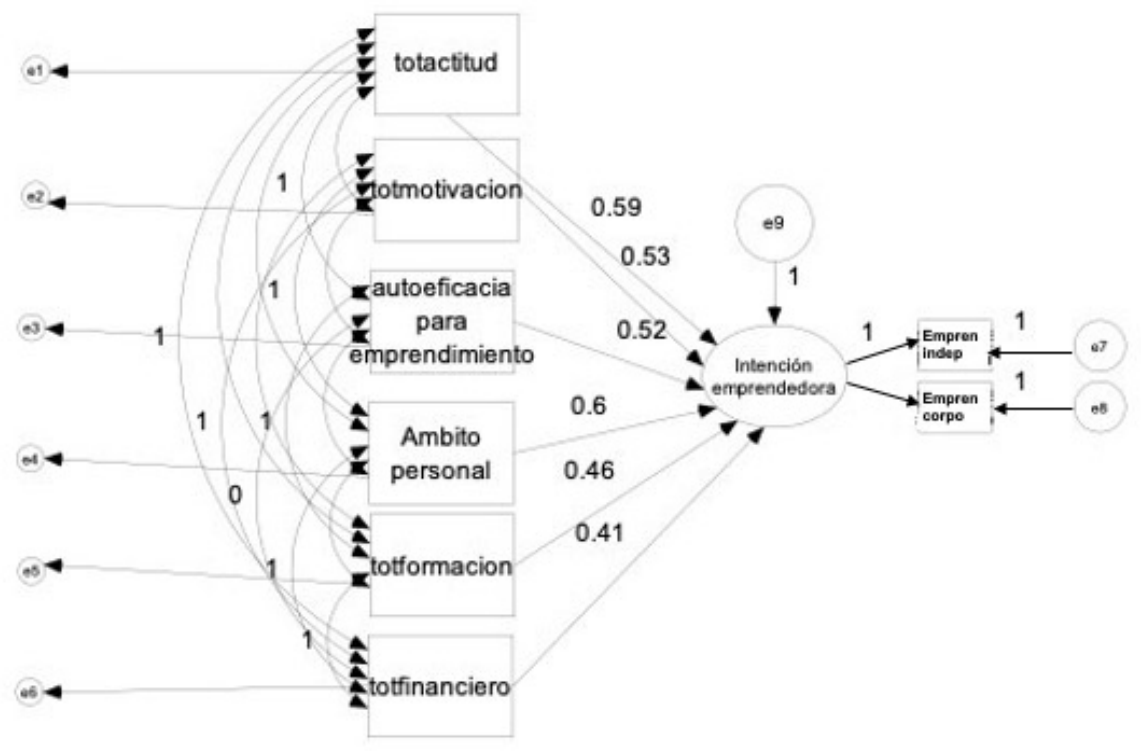

Figura 1. Primer modelo relación de todas las variables con la intención emprendedora.

En el siguiente modelo (modelo 2) ya sin estás variables los resultados indicaron todavía un pobre ajuste, con una X2 demasiado grande e indicadores de ajuste absoluto e incremental fuera de los valores de referencia retomados de Ruiz et al. ${ }^{52}$

Ante dicha situación se eliminó el elemento de apoyo financiero que se identificó tenía el estimado más fuera de rango, de tal forma que se probó el modelo 3 sin esta variable que tampoco obtuvo indicadores de ajuste adecuados, a la postre se eliminó la autoeficacia que era la variable con el estimado fuera de rango, de tal manera que en dicho modelo sólo quedaron la actitud emprendedora y la motivación para crear empresa para explicar la intención emprendedora, lo cual equivale al modelo 4, que se muestra en la figura 2. Este modelo ya presentó indicadores de ajuste más cercanos a los valores de referencia, comenzando por una X2 mayor de .01 aunque todavía con un RMR elevado.

52. Miguel A. Ruiz, Antonio Pardo y Rafael San Martín, "Modelos de ecuaciones estructurales”. Papeles del Psicólogo 31,1 (2010): 34-45. 


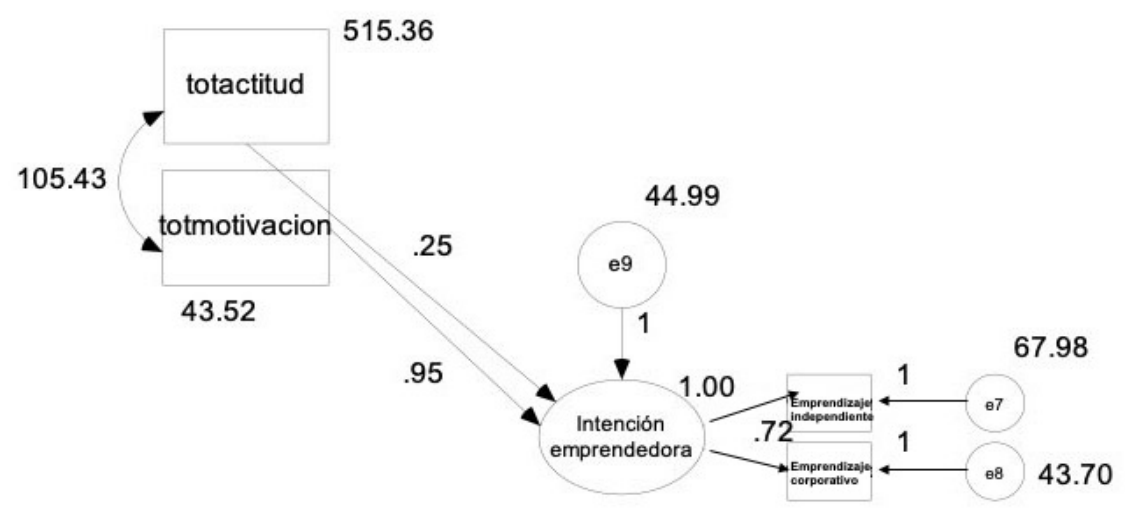

Figura 2. Cuarto modelo actitud y motivación y la intención emprendedora

Finalmente se realizó el último modelo que eliminaba la actitud emprendedora y dejaba sólo la motivación, que resultó ser la variable que mejor predice y explica la intención emprendedora, el quinto modelo puede verse en la figura 3, y en la tabla los indicadores de ajuste muestran que son también cercanos a la referencia y con menores residuos cuadráticos.

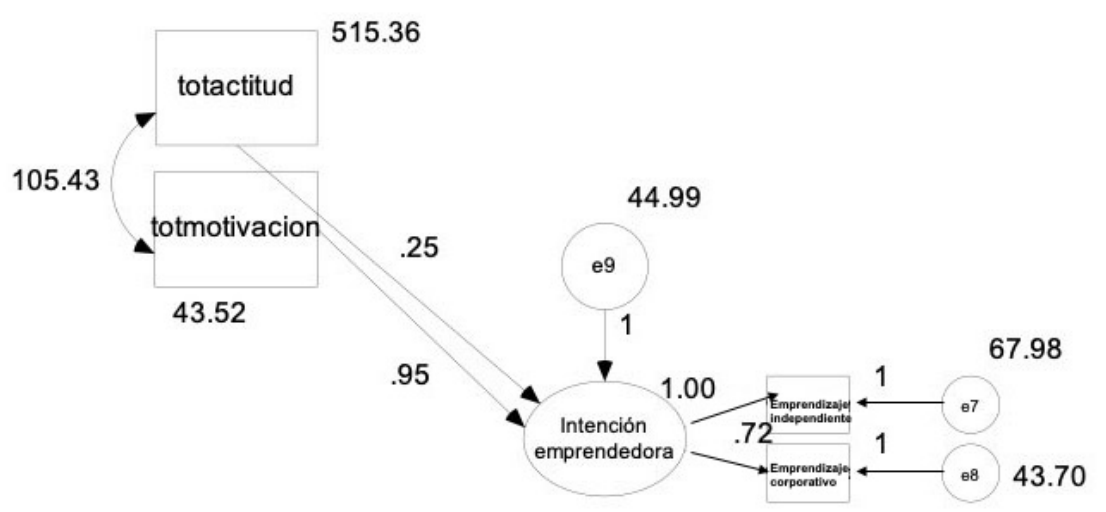

Figura 3. Quinto modelo motivación y emprendimiento.

Este último modelo mostró ser el que mejor ajusta, se debe tener en cuenta que se trata de una solución estadística que se acerca mejor a explicar la intención emprendedora y que muestra índices adecuados. 


\section{Discusión y Conclusiones}

El propósito central de este trabajo fue identificar la relación explicativa de la intención emprendedora, para ello se evaluaron diversos factores individuales que pueden influir en la intención de emprender; en relación a la autoeficacia que presentan los participantes respecto al emprendimiento, es decir que tanto cree la persona que cuenta con habilidades para desempeñar tareas relacionadas con la creación de una empresa, asimismo, aun cuando se ha identificado en algunos trabajos empíricos que la autoeficacia puede relacionarse con la intención de emprender, en esta investigación no es la variable predictora, Shane $\&$ Venkataraman (2003, Op.cit, 5p), apunta que una cuestión importante para que la autoeficacia sea determinante en el emprendimiento son las emociones, es decir aquel individuo que las maneja o controla sus emociones, sabe cómo identificarlas y emplea cada una de ellas de la mejor manera entonces tendrá mayor autoeficacia para emprender.

Aunque en otras investigaciones (Rueda, Henández \& Herrero, 2013, Op.cit, 7p) la actitud emprendedora es la que mejor predice la intención de emprender, es importante señalar que se deben tomar en cuenta las características de la muestra debido a que el grueso de esta muestra es de semestres básicos en los cuáles aún no tienen contacto directo con el campo de trabajo, a menos que se encuentren laborando, sin embargo sólo el $23 \%$ de los participantes trabaja, de tal manera que en una población que ya se encuentre laborando será más propenso a tener una actitud emprendedora.

La motivación para la creación de empresa en este estudio, es la variable que mejor predice y explica la intención emprendedora, lo cual coincide con Barba-Sánchez \& Atienza-Sahuquillo ${ }^{53}$ al encontrar en su investigación que la motivación impulsa a iniciar nuevos negocios, siendo una de las características el poder en el comportamiento empresarial, que es un aspecto que se relaciona con el indicador de estatus en la variable motivación en esta investigación. Sastre ${ }^{54}$ resalta que la motivación intrínseca en un inicio impulsa al emprendedor, pero al adquirir experiencia este, el factor extrínseco se vuelve más importante. Para Baena-Luna, García-Río y Monge-Agüero ${ }^{55}$ la motivación y perseverancia son aristas importantes al emprender, porque esto implica el convertir ideas en acción.

53. Virginia Barba-Sánchez and Carlos Atienza-Sahuquillo. "Entrepreneurial behavior: Impact of motivation factors on decision to create a new venture", Investigaciones Europeas de Dirección y Economía de la Empresa 18,2 (2012): 132-138.

54. Raquel F. Sastre, "La motivación emprendedora y los factores que contribuyen con el éxito del emprendimiento", Ciencias administrativas 1 (2013): 1-10.

55. Pedro Baena-Luna, Esther García-Río and Mauricio Monge-Agüero. "Entrecomp: marco competencial para el emprendimiento. Una revisión sistemática de la literatura sobre su uso y aplicación”. Información Tecnológica 31,2 (2020): $163-172$. 
Para próximas investigaciones se sugiere revisar los instrumentos que por su índice de confiabilidad presentan redundancia, lo que implica que existen ítems que miden lo mismo y pueden eliminarse, en otros estudios se debe tener una muestra similar de las diversas especialidades, ya que en estudios relacionados con la administración, informática tienen mayor formación emprendedora en comparación con especialidades en humanidades, sin embargo el no tener grupos con número equiparable no permite hacer estas comparaciones utilizando estadística paramétrica.

A manera de conclusión se puede señalar que la motivación es el factor más relevante para que un estudiante decida emprender, si bien también la actitud tiene peso sobre la intención de emprender, es importante señalar que ambos son factores personales del emprendedor.

\section{AGRADECIMIENTO}

Un agradecimiento y reconocimiento muy especial al profesor Fernando De Pasquale Arcia, Director de los Centros de Innovación, Desarrollo Tecnológico y Emprendimiento (CIDETE) de la Universidad de Panamá, por el apoyo y logística brindada para realizar el estudio en las diferentes sedes de la Universidad de Panamá.

También un agradecimiento a la Dra. Rosabel Alarcón Ramírez, Directora de la Dirección Universitaria de Educación a Distancia de la Universidad Alas Peruanas al autorizar la estancia de investigación y realizar el estudio sobre "Factores personales que influyen en la intención emprendedora en estudiantes universitarios” en la Universidad de Panamá.

\section{REFERENCIAS}

- $\quad$ Amit, R., and E. Muller. "Contrasting attributes and attitudes on entrepreneurs and nonentrepreneurs." Academy of Management National Meeting. 1994.

- Alonso Galicia, Patricia Esther. "La configuración de la intención emprendedora entre académicos responsables de proyectos de investigación en España. Un enfoque de género.” (2012).

- APA - American Psycgological Association. Manual de Publicaciones de la American Psychological Association. (3ra. Ed.) México: El Manual Moderno, (2010).

- $\quad$ Aragón, A. \& Baixauli, S. Intención Emprendedora de los estudiantes de Bachillerato y Ciclos Formativos en la Región de Murcia. Universidad de Murcia, 2014. 
- $\quad$ Azjen, Icek. "The theory of planned behavior." Organizational behavior and human decision processes 50.2 (1991): 179-211.

- Bandura, Albert. "Self-efficacy: toward a unifying theory of behavioral change." Psychological review 84.2 (1977): 191. https://doi.org/10.1037/0033-295X.84.2.191

- Baena-Luna, Pedro, García-Río, Esther and Monge-Agüero, Mauricio. "Entrecomp: marco competencial para el emprendimiento. Una revisión sistemática de la literatura sobre su uso y aplicación”. Información Tecnológica 31,2 (2020): 163-172.

http://dx.doi.org/10.4067/S0718-07642020000200163

- Barba-Sánchez, Virginia, and Carlos Atienza-Sahuquillo. "Entrepreneurial behavior: Impact of motivation factors on decision to create a new venture." Investigaciones Europeas de Dirección y Economía de la Empresa 18.2 (2012): 132-138.

http://dx.doi.org/10.1016/S1135-2523(12)70003-5

- Batalla, Fidel Rodriguez. Estudio de la intención emprendedora en el ámbito científico público. El caso de las ciencias de la vida en España. Diss. Universitat Politècnica de València, (2015).

- Bosma, Niels. "The Global Entrepreneurship Monitor (GEM) and its impact on entrepreneurship research.” Foundations and Trends in Entrepreneurship 9.2 (2013). https://doi.org/10.1561/0300000033

- Burgos, R. C. "Los emprendimientos como elemento de estudio teórico, académico e investigativo." Contribuciones a las Ciencias Sociales, Málaga, España, disponible en www. eumed. net/rev/cccss (2017).

- Cabana-Villca, Ricardo, et al. "Análisis de las capacidades emprendedoras potenciales y efectivas en alumnos de centros de educación superior."

Journal of technology management \& innovation 8.1 (2013): 65-75.

https://doi.org/10.4067/S0718-27242013000100007

- Castellanos Páez, Virgelina et al. "Modelo explicativo del desempeño académico desde la autoeficacia y los problemas de conducta." Revista Colombiana de psicología 26.1 (2017): 149-161. https://doi.org/10.15446/rcp.v26n1.56221 
- Castillo, Alicia. "Estado del arte en la enseñanza del emprendimiento." Emprendedores como creadores de riqueza y desarrollo regional 21 (1999).

- Cavazos-Arroyo, Judith, Rogelio Puente-Díaz, and Jannett Ayup-González. "Análisis de antecedentes de la intención al Emprendimiento social entre emprendedores potenciales de bajos ingresos en México". Vincula Tégica EFAN 2.1 (2016): 189-1915. Recuperado de: http://www.web.facpya.uanl.mx/Vinculategica/Revistas/R2/1889-1915\%20\%20 Analisis\%20De\%20Antecedentes\%20De\%20La\%20Intencion\%20Al\%20 Emprendimiento\%20Social\%20Entre\%20Emprendedores.pdf

- Cernas-Ortiz, Daniel Arturo, Patricia Mercado-Salgado, and Mark A. Davis. "Perspectiva futura de tiempo, satisfacción laboral y compromiso organizacional: el efecto mediador de la autoeficacia, la esperanza y la vitalidad." Revista de Psicología del Trabajo y de las Organizaciones 34.1 (2018): 1-9. https://doi.org/10.5093/jwop2018a1

- Correas, Clara Inés Orrego. "La dimensión humana del emprendimiento." Revista Ciencias Estratégicas 16.20 (2008): 225-236. Recuperado de: http://www.redalyc.org/html/1513/151312829001/

- Cupani, Marcos, "Análisis de ecuaciones estructurales: conceptos, etapas de desarrollo y un ejemplo de aplicación”. Revista Tesis 1, (2012): 186-199.

- Delgado-García, Juan Bautista, Rodríguez-Escudero, Ana Isabel, and Martín-Cruz, Natalia. "Influence of affective traits on entrepreneur's goals and satisfaction." Journal of Small Business Management 50.3 (2012): 408-428. https://doi.org/10.1111/j.1540-627X.2012.00359.x

- Del Río, Ainara Zubillaga. "El emprendimiento como elemento de formación universitaria: en busca de nuevas propuestas." Creatividad y sociedad: revista de la Asociación para la Creatividad 23 (2015): 182-199.

- Durán-Aponte, Emilse, and Arias-Gómez, Diana. "Intención emprendedora en estudiantes universitarios: integración de factores cognitivos y socio-personales." Revista Colombiana de Ciencias Sociales 6.2 (2015): 320-340. 
- Guerrero, Maribel y Santamaría-Velasco, Carlos Alberto. “Ecosistema y actividad emprendedora en México: un análisis exploratorio”, Perfiles Latinoamericanos, 28, 55 (2020): 227-251. https://doi.org/10.18504/pl2855-009-2020

- Hinojoza, Daniel Mavila, Tinoco Gómez, Óscar, and Campos Contreras, César. "Factores influyentes en la capacidad emprendedora de los alumnos de la Universidad Nacional Mayor de San Marcos." Industrial Data 12.2 (2009): 32-39.

- Izquierdo, Edgar. Impact assessment of an educational intervention based on the constructivist paradigm on the development of entrepreneurial competencies in university students. Diss. Ghent University, Faculty of Economics and Business Administration, 2008.

- Krauss, Catherine. "Actitudes emprendedoras de los estudiantes universitarios: El caso de la Universidad Católica del Uruguay.” Dimensión empresarial 9.1 (2011): 28-40.

- López, Juan Cordero, et al. "Análisis de los factores que influyen el emprendimiento y la sostenibilidad de las empresas del área urbana de la ciudad de Cuenca, Ecuador." Maskana 2.2 (2011): 27-37. https://doi.org/10.18537/mskn.02.02.03

- $\quad$ López, I. D., B. Santos, and Y. Bueno. "Las dimensiones del perfil del emprendedor: contraste empírico con emprendedores de éxito." Presentado en el congreso $E l$ emprendedor innovador y la creación de empresas de I+ D+ I. Universidad de Valencia: Valencia, España. 2004.

- Martínez-Rodríguez, Facundo Miguel, and Carmona Orantes, Gabriel. “Test de factores sociopersonales para la inserción laboral de los jóvenes: un instrumento para la evaluación y la formación.” Estudios sobre educación 18 (2010): 115-138.

- Marulanda Valencia, Flor A., Montoya Restrepo, Iván A. and Vélez Restrepo, Juan M. "Aportes teóricos y empíricos al estudio del emprendedor." Cuadernos de administración 30.51 (2014): 89-99. https://doi.org/10.25100/cdea.v30i51.46

- Moriano, Juan Antonio, Trejo, Enrique, and Palací, Francisco J. "El perfil psicosocial del emprendedor: un estudio desde la perspectiva de los valores.” Revista de psicología Social 16.2 (2001): 229-242. https://doi.org/10.1174/021347401317351152 
- Olmos, Roberto Espíritu, and Miguel Ángel Sastre Castillo. "La actitud emprendedora durante la vida académica de los estudiantes universitarios." Cuadernos de estudios empresariales 17 (2007): 95-116.

- Olmos, Roberto Espíritu. "Análisis de la intención emprendedora en estudiantes universitarios a través de los rasgos de personalidad." Multiciencias 11.1 (2011): 65-75.

- Oviedo, Heidi Celina and Campo-Arias, Adalberto. "Aproximación al uso del coeficiente alfa de Cronbach”, Revista Colombiana de Psiquiatría 34,4 (2005): 572-580

- Paredes, M. "Relación entre intención emprendedora, aspiraciones y satisfacción con la vida en universitarios limeños." Universidad Peruana de Ciencias Aplicadas. Lima-Perú (2015).

- Penteado Pedroso, José Pedro, Márcia Shizue Massukado-Nakatani, and Fabrício Baron Mussi. "A relação entre o jeitinho brasileiro e o perfil empreendedor: possíveis interfaces no contexto da atividade empreendedora no Brasil.” RAM. Revista de Administração Mackenzie 10.4 (2009): 100-130. https://doi.org/10.1590/S1678-69712009000400006

- $\quad$ Rasheed, Howard. "Developing Entrepreneutial Potencial in Youth of Entrepreneurial Education and Venture Creation". University of South Florida (2000).

- $\quad$ Ronstadt, Robert, Karl H. Vesper, and W. Ed McMullan. "Entrepreneurship: today courses, tomorrow degrees?." Entrepreneurship theory and practice 13.1 (1988): 7-13.

https://doi.org/10.1177/104225878801300102

- $\quad$ Rueda Sampedro, María Inés, Ana Fernández Laviada, and Angel Herrero Crespo. "Aplicación de la teoría de la acción razonada al ámbito emprendedor en un contexto universitario." (2013).

- $\quad$ Ruiz, Miguel A., Pardo, Antonio y San Martín, Rafael, "Modelos de ecuaciones estructurales". Papeles del Psicólogo 31,1 (2010): 34-45.

- Sastre, Raquel F. "La motivación emprendedora y los factores que contribuyen con el éxito del emprendimiento." Ciencias administrativas 1 (2013): 1-10.

- Solomon, George, et al. "Entrepreneur's profile: a taxonomy of attributes and motivations of university students." Journal of Small Business and enterprise development (2008). https://doi.org/10.1108/14626000810871763 
- Solomon, Michael R., and Miguel Angel Sanchez Carrion. Comportamiento del consumidor. Vol. 12. Pearson, 2017.

- Sorondo, J., y M. E. Brenlla. "Locus de control y autoeficacia en emprendedores argentinos". Trabajo de integración final, Universidad católica argentina (2011).

- Schwartz, Shalom H., and Wolfgang Bilsky. "Toward a theory of the universal content and structure of values: Extensions and cross-cultural replications." Journal of personality and social psychology 58.5 (1990): 878. https://doi.org/10.1037/0022-3514.58.5.878

- Shafritz, Jay M., J. Ott, Steven and Yong Suk Jang. Classics of organization theory.Cengage Learning, 2015.

- Shane, Scott, Edwin A. Locke, and Christopher J. Collins. "Entrepreneurial motivation." Human resource management review 13.2 (2003): 257-279.

- Shane, Scott, and Sankaran Venkataraman. "The promise of entrepreneurship as a field of research.” Entrepreneurship. Springer, Berlin, Heidelberg, 2007. 171-184.

https://doi.org/10.5465/amr.2000.2791611

- Shapero, Albert. "Why entrepreneurship? A worldwide perspective.” Journal of Small Business Management (pre-1986) 23.000004 (1985): 1.

- Urbano, David, and Nuria Toledano Garrido. "Los sistemas de formación universitaria y su influencia en las actitudes empresariales de los estudiantes: Un estudio de casos múltiple." Oikos: Revista de la Escuela de Administración y Economía 25 (2008): 5.

- Valbuena Hernández, Pedro Nel and Borda Hernández, Ricardo Alberto. "Características emprendedoras en estudiantes de la Universidad El Bosque (Colombia)”. Revista Espacios 38,30 (2017):22-30.

- Vásquez, Samuel. "Intención emprendedora en estudiantes universitarios de Ingeniería Industrial Administrativa de la Universidad Católica Santa María la Antigua (USMA).” Investigación y Pensamiento Crítico 3.3 (2015): 13-26.

https://doi.org/10.37387/ipc.v3i3.54 
- Veciana, José M. La creación de empresas: Un enfoque gerencial. Caixa, Servicio de Estudios, 2005.

- Zabala, R. (2013). "Emprendimiento: un proceso de aprendizaje”. Recuperado de http://www.madrimasd.org/informacionIdi/analisis/opinion/opinion.asp?id=55885

RECIBIDO: $14 / 03 / 2020$

APROBADO: $20 / 05 / 2020$ 